\title{
A Block Cipher Involving a Key Bunch Matrix and an Additional Key Matrix, Supplemented with XOR Operation and Supported by Key-Based Permutation and Substitution
}

\author{
Dr. V.U.K.Sastry \\ Professor (CSE Dept), Dean (R\&D) \\ SreeNidhi Institute of Science \& Technology, SNIST \\ Hyderabad, India
}

\begin{abstract}
In this paper, we have developed a block cipher by extending the analysis of a Novel Block Cipher Involving a Key bunch Matrix and a Key-based Permutation and Substitution. Here we have include and additional key matrix, which is supplemented with xor operation. The cryptanalysis carried out in this investigation clearly indicates that this cipher cannot be broken by any attack.
\end{abstract}

Keywords- Key; key bunch matrix; encryption; decryption; permutation; substitution; avalanche effect; cryptanalysis; xor operation

\section{INTRODUCTION}

In a recent investigation [1], we have developed a block cipher involving a key bunch matrix and including a pair of functions, called Permute() and Substitute(). In this analysis, we have seen that the permutation and the substitution, which depend effectively on a key, strengthen the cipher in a remarkable manner. This is all on account of the fact that the permutation and the substitution, induced into the plaintext at each and every stage in the iteration process, causes confusion and diffusion.

In the present investigation, our objective is to modify the afore-mentioned block cipher by introducing an additional key matrix supplemented with xor operation. The basic equation governing the encryption of this cipher can be written in the form

$$
\mathrm{C}=\left[c_{i j}\right]=\left(\left[e_{i j} \times p_{i j}\right] \bmod 256\right) \oplus \mathrm{F}, \mathrm{i}=1 \text { to } \mathrm{n}, \mathrm{j}=1
$$
to $\mathrm{n}$.

The corresponding equation describing decryption can be written in the form

$$
\mathrm{P}=\left[p_{i j}\right]=\left[d_{i j} \times(\mathrm{C} \oplus \mathrm{F})_{i j}\right] \bmod 256, \mathrm{i}=1 \text { to } \mathrm{n}, \mathrm{j}=1 \text { to }
$$

n.

Here, our interest is to examine, how the additional key matrix, F, would strengthen the cipher when supported by permuted and substitution.

\author{
K. Shirisha \\ Computer Science \& Engineering \\ SreeNidhi Institute of Science \& Technology, SNIST \\ Hyderabad, India
}

Let us now present the plan of the paper. In section 2, we introduce the development of the cipher. Here, we depict the flowcharts and write the algorithms required in this investigation. Then, we mention the basic ideas of the key based permutation and substitution. In section 3, we mention an illustration of the cipher, and discuss the avalanche effect. We study the cryptanalysis, in section 4 . Finally, we deal with the computations carried out in this analysis, and draw conclusions, in section 5 .

\section{DEVELOPMENT OF THE CIPHER}

Consider a plaintext, which can be written in the form of a square matrix $\mathrm{P}$, given by

$$
\mathrm{P}=\left[p_{i j}\right], \mathrm{i}=1 \text { to } \mathrm{n}, \mathrm{j}=1 \text { to } \mathrm{n} \text {. }
$$

Let us take a key bunch matrix $\mathrm{E}$, given by

$$
\mathrm{E}=\left[e_{i j}\right], \mathrm{i}=1 \text { to } \mathrm{n}, \mathrm{j}=1 \text { to } \mathrm{n} \text {. }
$$

On using the concept of the multiplicative inverse [2], we get $d_{i j}$ corresponding to each $e_{i j}$. Thus we have the decryption key bunch matrix $\mathrm{D}$, given by

$$
\mathrm{D}=\left[d_{i j}\right], \mathrm{i}=1 \text { to } \mathrm{n}, \mathrm{j}=1 \text { to } \mathrm{n} .
$$

Here, it is to be noted that, all the $e_{i j}$ and $d_{i j}$ are odd numbers which lie in the interval [1-255].

The flowcharts concerned to the encryption and the decryption are drawn in Figs. 1 and 2.

The corresponding algorithms for the encryption and the decryption are as follows.

$$
\begin{aligned}
& \text { Algorithm for Encryption } \\
& \text { 1. } \text { Read } P, E, K, F, n, r \\
& \text { 2. } \text { For } \mathrm{k}=1 \text { to } \mathrm{r} \text { do } \\
&\{\text { } \\
& \text { 3. } \text { For } \mathrm{i}=1 \text { to } \mathrm{n} \text { do } \\
&\{ \\
& \text { 4. } \text { For } \mathrm{j}=1 \text { to } \mathrm{n} \text { do } \\
&\{
\end{aligned}
$$



\}
\}
6. $\mathrm{P}=\left[p_{i j}\right] \oplus \mathrm{F}$
7. $\mathrm{P}=$ Permute $(\mathrm{P})$
8. $\mathrm{P}=$ Substitute $(\mathrm{P})$ \}
8. $\mathrm{C}=\mathrm{P}$
9. Write $(\mathrm{C})$

5. $\quad p_{i j}=\left(e_{i j} \times p_{i j}\right) \bmod 256$

\section{Algorithm for Decryption}

1. Read C,E,K,F,n,r

2. $\mathrm{D}=\mathrm{Mult}(\mathrm{E})$

3. For $\mathrm{k}=1$ to $\mathrm{r}$ do \{

4. $\mathrm{C}=\mathrm{ISubstitute}(\mathrm{C})$

5. $\mathrm{C}=$ IPermute $(\mathrm{C})$

6. For $\mathrm{i}=1$ to $\mathrm{n}$ do \{

7. For $\mathrm{j}=1$ to $\mathrm{n}$ do \{

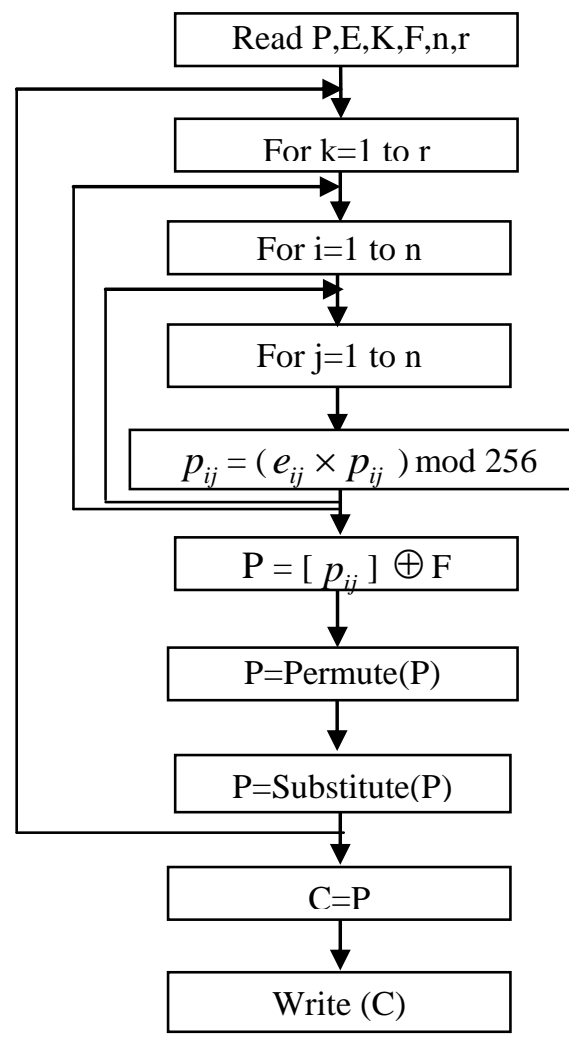

Fig.1 Flowchart for Encryption
8. $c_{i j}=\left[d_{i j} \times\left(c_{i j} \oplus f_{i j}\right)\right] \bmod 256$

$$
\text { \} }
$$

9. $\mathrm{C}=\left[c_{i j}\right]$<smiles>C1CCC1</smiles>

10. $\mathrm{P}=\mathrm{C}$

11. Write $(\mathrm{P})$

In this analysis, $r$ denotes the number of rounds in the iteration process, and it is taken as 16 .

The functions Permute() and Substitute(), which are utilized in encryption, depend upon a key. Let us choose the $\mathrm{key}, \mathrm{K}$, in the form

$$
K=\left[\begin{array}{cccc}
156 & 14 & 33 & 96 \\
253 & 107 & 110 & 127 \\
164 & 10 & 5 & 123 \\
174 & 202 & 150 & 94
\end{array}\right]
$$

Keeping the serial numbers and the order of the elements in the key, in view, we construct a table of the form given in Table-1.

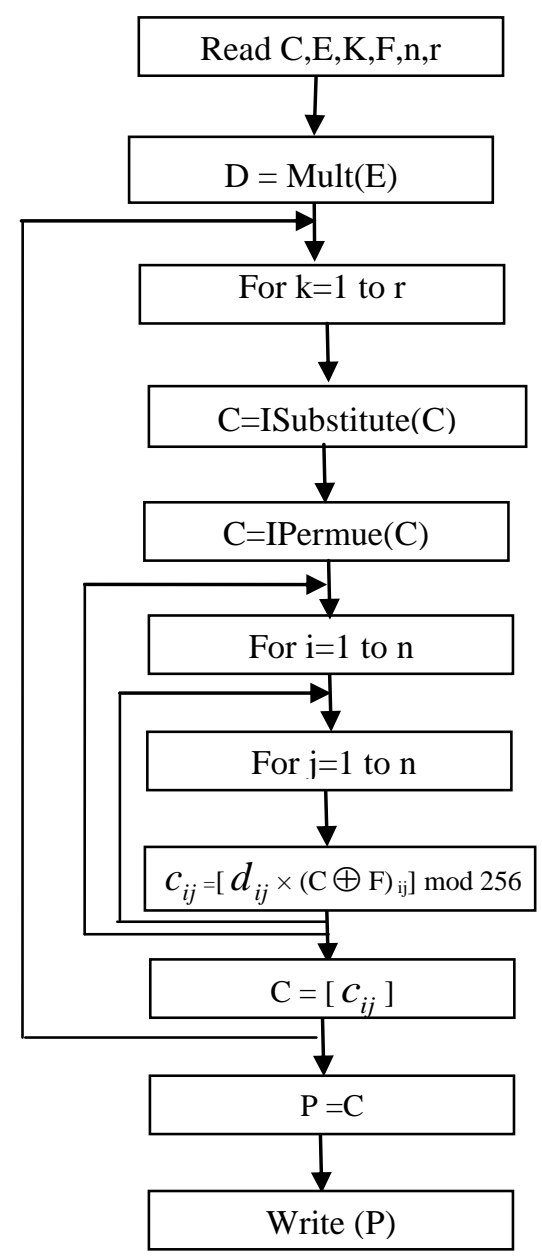

Fig.2 Flowchart for Decryption 
Table-1. Relation Between Serial Numbers AND Numbers In Ascending ORder.

\begin{tabular}{|r|r|r|r|r|r|r|r|r|r|r|r|r|r|r|r|}
\hline 1 & 2 & 3 & 4 & 5 & 6 & 7 & 8 & 9 & 10 & 11 & 12 & 13 & 14 & 15 & 16 \\
\hline 156 & 14 & 33 & 96 & 253 & 107 & 110 & 127 & 164 & 10 & 5 & 123 & 174 & 202 & 150 & 94 \\
\hline 12 & 3 & 4 & 6 & 16 & 7 & 8 & 10 & 13 & 2 & 1 & 9 & 14 & 15 & 11 & 5 \\
\hline
\end{tabular}

The process of permutation can be explained as follows.

Let $\mathrm{x}_{\mathrm{i}}, \mathrm{i}=1$ to 16 , be a set of 16 numbers. As the table is suggesting (looking at the first row and the third row), we interchange $x_{1}$ with $x_{12}, x_{2}$ with $x_{3}, x_{4}$ with $x_{6}, x_{5}$ with $x_{16}, x_{7}$ with $x_{8}, x_{9}$ with $x_{13}$, and $x_{14}$ with $x_{15}$. It may be noted here that we need not interchange any other numbers as they are already subjected to change in a way. Keeping this basic idea in view, let us now consider the plaintext matrix $\mathrm{P}=$ [ $p_{i j}$ ], $\mathrm{i}=1$ to $\mathrm{n}, \mathrm{j}=1$ to $\mathrm{n}$, (after xoring with $\mathrm{F}$ ) in any round of the iteration process. Considering the first two rows of this matrix and representing the elements $p_{i j}$ in their binary form, and writing the binary bits in the vertical manner, we get a matrix of size $16 \times n$. On dividing this matrix into submatrices, where each one of size 16x16, and performing the interchange of rows (firstly) and columns (subsequently), as is done in the case of numbers $x_{i}, \mathrm{i}=1$ to 16 , we get the corresponding permuted matrix, in the case of each submatrix. On applying the same procedure for the other submatrices also, we ultimately get n/16 sub-matrices. On representing the binary bits in terms of decimal numbers (converting 8 binary bits in a row as a decimal number), we get a $2 x n$ matrix. On adopting the same procedure on the subsequent pairs of this matrix, we complete the permutation process. However, it is to be remembered that $n$ must be divisible by 16 . In case, if $n<16$, that is say, $n=4$, then a plaintext matrix of size $4 \times 4$ can be written as a matrix of size $8 \times 16$, by writing each decimal number as binary bits in a column. Then the procedure of swapping, applied for numbers, can be applied here, for rows firstly and for columns nextly.

However, in the case of rows, we restrict our interchanging process only to 8 rows. Then, on representing the binary bits in terms of decimal numbers (considering the bits in a rowwise manner) we get the permuted matrix. This completes the process of permutation.

The process of substitution can be mentioned as follows. In the EBCDIC code, the characters can be represented in terms of a table of size $16 \times 16$, containing numbers 0 to 255 , in a sequential manner. On swapping rows, firstly, and columns, nextly, as it is already done in the case of the numbers $x_{1}$ to $x_{16}$, we get a new table (see Table-2).

On using the Table-2, we perform substitution, by noting the correspondence between the number in the plaintext, the number in the EBCDIC table and hence the number in the substitution table. For clarity if this substitution process, we refer to [1].

The functions IPermute() and ISubstitute(), used in the decryption process, denote the reverse processes of the Permute() and the Substitute(). The function Mult() is used to find the decryption key bunch matrix $\mathrm{D}$ for the given $\mathrm{E}$.

\section{Illustration OF THE CIPHER AND THE AVALANCHE EFFECT}

Consider the plaintext given below.

Dear Madam! I have received your letter. Please do not run away from our country in that manner. I am coming within this month. I will not continue my Ph.D. programme. I may leave this research activity but I cannot leave you. It is indeed a surprise. Though there was no response from the selection committee for a span of one year, very recently I got selected in our country for IAS. I think I am lucky. Tell you father and mother about this news and tell them in a nice manner that you are running p8third month. I hope that all these issues will end up very soon and we will become one undoubtedly. Tell my father and mother that I am coming there. Yours loving husband

Let us focus our attention on the first 16 characters of this plaintext. Thus we have

\section{Dear Madam! I ha}

On using the EBCDIC code, we get

$$
P=\left[\begin{array}{cccc}
196 & 133 & 129 & 153 \\
64 & 212 & 129 & 132 \\
129 & 148 & 79 & 64 \\
201 & 64 & 136 & 129
\end{array}\right] .
$$

Let us take the key bunch matrix $\mathrm{E}$ in the form

$$
E=\left[\begin{array}{cccc}
199 & 23 & 67 & 211 \\
67 & 91 & 93 & 5 \\
11 & 19 & 51 & 145 \\
109 & 223 & 251 & 5
\end{array}\right] .
$$

On using the concept of the multiplicative inverse, we have the decryption key bunch matrix $\mathrm{D}$ in the form

$$
D=\left[\begin{array}{cccc}
247 & 167 & 107 & 91 \\
107 & 211 & 245 & 205 \\
163 & 27 & 251 & 113 \\
101 & 31 & 51 & 205
\end{array}\right]
$$




\begin{tabular}{|c|c|c|c|c|c|c|c|c|c|c|c|c|c|c|c|}
\hline 187 & 178 & 177 & 181 & 191 & 179 & 183 & 182 & 188 & 185 & 186 & 176 & 184 & 190 & 189 & 180 \\
\hline 43 & 34 & 33 & 37 & 47 & 35 & 39 & 38 & 44 & 41 & 42 & 32 & 40 & 46 & 45 & 36 \\
\hline 27 & 18 & 17 & 21 & 31 & 19 & 23 & 22 & 28 & 25 & 26 & 16 & 24 & 30 & 29 & 20 \\
\hline 91 & 82 & 81 & 85 & 95 & 83 & 87 & 86 & 92 & 89 & 90 & 80 & 88 & 94 & 93 & 84 \\
\hline 51 & 242 & 241 & 245 & 255 & 243 & 247 & 246 & 252 & 249 & 250 & 240 & 248 & 254 & 253 & 244 \\
\hline 59 & 50 & 49 & 53 & 63 & 51 & 55 & 54 & 60 & 57 & 58 & 48 & 56 & 62 & 61 & 52 \\
\hline 123 & 114 & 113 & 117 & 127 & 115 & 119 & 118 & 124 & 121 & 122 & 112 & 120 & 126 & 125 & 116 \\
\hline 107 & 98 & 97 & 101 & 111 & 99 & 103 & 102 & 108 & 105 & 106 & 96 & 104 & 110 & 109 & 100 \\
\hline 203 & 194 & 193 & 197 & 207 & 195 & 199 & 198 & 204 & 201 & 202 & 192 & 200 & 206 & 205 & 196 \\
\hline 155 & 146 & 145 & 149 & 159 & 147 & 151 & 150 & 156 & 153 & 154 & 144 & 152 & 158 & 157 & 148 \\
\hline 171 & 162 & 161 & 165 & 175 & 163 & 167 & 166 & 172 & 169 & 170 & 160 & 168 & 174 & 173 & 164 \\
\hline 11 & 2 & 1 & 5 & 15 & 3 & 7 & 6 & 12 & 9 & 10 & 0 & 8 & 14 & 13 & 4 \\
\hline 139 & 130 & 129 & 133 & 143 & 131 & 135 & 134 & 140 & 137 & 138 & 128 & 136 & 142 & 141 & 132 \\
\hline 235 & 226 & 225 & 229 & 239 & 227 & 231 & 230 & 236 & 233 & 234 & 224 & 232 & 238 & 237 & 228 \\
\hline 219 & 210 & 209 & 213 & 223 & 211 & 215 & 214 & 220 & 217 & 218 & 208 & 216 & 222 & 221 & 212 \\
\hline 75 & 66 & 65 & 69 & 79 & 67 & 71 & 70 & 76 & 73 & 74 & 64 & 72 & 78 & 77 & 68 \\
\hline
\end{tabular}

TABLE-2 Key BASEd Substitution

The additional key matrix $\mathrm{F}$ is taken in the form

$$
F=\left[\begin{array}{llll}
222 & 243 & 122 & 45 \\
56 & 22 & 100 & 99 \\
104 & 76 & 45 & 11 \\
9 & 22 & 25 & 67
\end{array}\right] .
$$

Now, on making use of the plaintext $\mathrm{P}$, the encryption key bunch matrix $\mathrm{E}$ and the additional key matrix $\mathrm{F}$, and applying the encryption algorithm, given in section 2, we get the ciphertext matrix $\mathrm{C}$ in the form

$$
C=\left[\begin{array}{cccc}
88 & 2 & 165 & 241 \\
47 & 226 & 95 & 110 \\
214 & 121 & 129 & 163 \\
104 & 97 & 195 & 215
\end{array}\right] .
$$

On using this $\mathrm{C}$, the $\mathrm{F}$, and the decryption key bunch matrix $\mathrm{D}$, given by (3.5), and the decryption algorithm, given in section 2 , we get back the plaintext $\mathrm{P}$, which is in the form (3.3).

Now let us study the avalanche effect. On replacing the 4th row 4th column element, 129 in the plaintext (3.3) by 193, we have a one binary bit change in the plaintext. On using this modified plaintext, the $\mathrm{E}$, the $\mathrm{F}$, and the encryption algorithm, we get the new ciphertext in the form, given by (3.8).

On comparing (3.8) and (3.7), after converting them into their binary form, we notice that there is a change of 72 bits out of 128 bits. This shows that the cipher is a strong one.

$$
C=\left[\begin{array}{cccc}
1 & 198 & 243 & 34 \\
189 & 43 & 134 & 140 \\
89 & 195 & 102 & 168 \\
149 & 148 & 254 & 196
\end{array}\right] .
$$

Now, let us have one binary bit change in the key bunch matrix E. To this end, we replace the 3rd row 2 nd column element 19 in $\mathrm{E}$ by 18 . On using this modified $\mathrm{E}$, the original $\mathrm{P}$, given by (3.3), and the F, given by (3.6), and applying the encryption algorithm, we get the corresponding ciphertext $\mathrm{C}$, in the form

$$
C=\left[\begin{array}{cccc}
154 & 160 & 102 & 158 \\
173 & 29 & 134 & 243 \\
236 & 190 & 127 & 195 \\
209 & 188 & 48 & 241
\end{array}\right] .
$$

Now, let us convert (3.7) and (3.9) into their binary form and compare them. From this, we find that these two ciphertexts differ by 74 bits out of 128 bits. This also shows that the cipher is having appreciable strength.

Now, on making use of the plaintext $\mathrm{P}$, the encryption key bunch matrix $\mathrm{E}$ and the additional key matrix $\mathrm{F}$, and applying the encryption algorithm, given in section 2, we get the ciphertext matrix $\mathrm{C}$ in the form

On using this $\mathrm{C}$, the $\mathrm{F}$, and the decryption key bunch matrix D, given by (3.5), and the decryption algorithm, given in section 2 , we get back the plaintext $\mathrm{P}$, which is in the form (3.3).

Now let us study the avalanche effect. On replacing the 4th row 4th column element, 129 in the plaintext (3.3) by 193, we have a one binary bit change in the plaintext. On using this modified plaintext, the $\mathrm{E}$, the $\mathrm{F}$, and the encryption algorithm, we get the new ciphertext in the form

Now, let us convert (3.7) and (3.9) into their binary form and compare them. From this, we find that these two ciphertexts differ by 74 bits out of 128 bits. This also shows that the cipher is having appreciable strength.

\section{CRYPTANALYSIS}

The study of cryptanalysis plays a prominent role in the development of every cipher. The different types of attacks that are available in the literature of cryptography are

1. Ciphertext only attack (Brute force attack),

2. Known plaintext attack,

3. Chosen plaintext attack, and

4. Chosen ciphertext attack.

Generally, every algorithm is designed [2] such that it withstands the first two attacks. The cipher is also examined in a thorough manner, by using all possible intuitive ideas, in the case of the latter two attacks. 
In this analysis, we have the key bunch matrix E, whose size is nxn. Besides this, we have the additional key matrix F, whose size is also nxn. In addition to these two, we have the key matrix $\mathrm{K}$ which is used in the development of permutation and substitution processes. In view of all these three, the size of the key space is

$$
\begin{aligned}
& 2^{7 n^{2}} \times 2^{8 n^{2}} \times 2^{128}=2^{7 n^{2}+8 n^{2}+128}=2^{15 n^{2}+128} \\
& =\left(2^{10}\right)^{\left(1.5 n^{2}+12.8\right)} \approx\left(10^{3}\right)^{\left(1.5 n^{2}+12.8\right)}=10^{4.5 n^{2}+38.4}
\end{aligned}
$$

On assuming that, we require $10^{-7}$ seconds for computation with one set of-n keys in the key space, then the time required for all such possible set $\mathrm{s}$ in the key space is

$$
\frac{10^{4.5 n^{2}+38.4} \times 10^{-7}}{365 \times 24 \times 60 \times 60}=3.12 \times 10^{4.5 n^{2}+23.4} \text { years } \text {. }
$$

In our present analysis, as $n=4$, the time for computation with all possible sets of keys in the key space is

$$
3.12 \times 10^{95.4} \text { years. }
$$

As this time is very large, it is simply impossible to break this cipher by the brute force attack.

Let us now examine the known plaintext attack. In order to carry out this approach, we have plaintext and ciphertext pairs, as many as we want, at our disposal. If we focus our attention on only one round of the iteration process, that is if $r=1$, then the basic equations governing the encryption process are given by

$$
\begin{aligned}
& \mathrm{P}=\left(\left[e_{i j} \times p_{i j}\right] \bmod 256\right) \oplus \mathrm{F}, \mathrm{i}=1 \text { to } \mathrm{n}, \mathrm{j}=1 \text { to } \mathrm{n}, \\
& \mathrm{P}=\operatorname{Permute}(\mathrm{P}), \\
& \mathrm{P}=\operatorname{Substitute}(\mathrm{P}), \\
& \text { and } \\
& \mathrm{C}=\mathrm{P}
\end{aligned}
$$

In this attack, the ciphertext $\mathrm{C}$ in (4.4), is known to us. On using this one, we can know the $\mathrm{P}$, occurring in the left side of (4.3). As key is unknown, we do not know ISubstitute(). Hence $\mathrm{P}$ occurring on the right hand side of (4.3) cannot be determined. Hence this cipher cannot be broken by the known plaintext attack. Luckily, if key $\mathrm{K}$ is known (a very stray case), then we can obtain $\mathrm{P}$, occurring on the left hand side of (4.1). Then, though $p_{i j}$ is known to us, we cannot determine the $e_{i j}$, by any means, as the equation (4.1) is containing several unknowns related to $\mathrm{F}$, and is including mod and xor operations. Thus this cipher cannot be broken by the known plaintext attack, even when $\mathrm{r}=1$, and the key matrix $\mathrm{K}$, used in the permutation process is known to the attacker.

In view of the equations, involved in the encryption process, we do not find any possibility to choose either a plaintext or a ciphertext for breaking this cipher.

In the light of the above facts, we conclude that this cipher is a very strong one.

\section{COMPUTATIONS AND CONCLUSIONS}

In this investigation, we have developed a block cipher involving a key bunch matrix and an additional key matrix, and involving key-based permutation and substitution. The strength of the cipher is highly remarkable due to permutation and substitution, and it is further supplemented with the additional key matrix. Java.

The programs for encryption and decryption are written in

In order to carry out the encryption of the entire plaintext, given by (3.1), we use a large size encryption key bunch matrix EK of size 16x16. Along with this, we have taken an additional key matrix FK, which is also of the same size 16x16. The EK and FK are given below in (5.1) and (5.2).

$E K=\left[\begin{array}{cccccccccccccccc}125 & 171 & 129 & 101 & 141 & 225 & 251 & 47 & 69 & 123 & 121 & 65 & 177 & 5 & 131 & 243 \\ 213 & 29 & 227 & 127 & 61 & 107 & 195 & 145 & 83 & 89 & 221 & 167 & 151 & 79 & 125 & 167 \\ 3 & 41 & 213 & 161 & 35 & 131 & 203 & 125 & 125 & 41 & 177 & 231 & 15 & 21 & 93 & 111 \\ 209 & 83 & 65 & 203 & 183 & 163 & 165 & 59 & 123 & 15 & 113 & 157 & 249 & 243 & 171 & 113 \\ 195 & 45 & 63 & 23 & 191 & 197 & 25 & 129 & 177 & 151 & 221 & 217 & 21 & 173 & 31 & 185 \\ 103 & 17 & 47 & 3 & 223 & 223 & 167 & 13 & 43 & 241 & 173 & 117 & 31 & 113 & 227 & 93 \\ 37 & 219 & 195 & 175 & 199 & 83 & 79 & 217 & 233 & 217 & 169 & 253 & 127 & 75 & 163 & 243 \\ 215 & 111 & 79 & 159 & 193 & 5 & 231 & 117 & 55 & 55 & 63 & 119 & 249 & 205 & 193 & 13 \\ 231 & 243 & 199 & 115 & 201 & 67 & 173 & 195 & 19 & 191 & 17 & 145 & 219 & 155 & 39 & 241 \\ 251 & 223 & 231 & 95 & 105 & 201 & 119 & 51 & 181 & 229 & 181 & 167 & 247 & 153 & 225 & 149 \\ 37 & 183 & 253 & 177 & 117 & 33 & 17 & 231 & 163 & 83 & 195 & 157 & 223 & 13 & 95 & 95 \\ 183 & 241 & 95 & 53 & 247 & 117 & 169 & 23 & 27 & 107 & 85 & 167 & 215 & 171 & 203 & 139 \\ 49 & 221 & 127 & 69 & 127 & 245 & 73 & 3 & 113 & 125 & 237 & 45 & 55 & 115 & 241 & 221 \\ 213 & 85 & 21 & 15 & 21 & 205 & 85 & 203 & 105 & 235 & 155 & 5 & 105 & 153 & 109 & 135 \\ 223 & 133 & 239 & 181 & 127 & 157 & 77 & 243 & 17 & 129 & 133 & 161 & 11 & 65 & 93 & 169 \\ 91 & 59 & 171 & 201 & 53 & 91 & 31 & 169 & 203 & 113 & 181 & 125 & 151 & 165 & 245 & 51\end{array}\right]$

and 


$$
F K=\left[\begin{array}{cccccccccccccccc}
91 & 46 & 145 & 165 & 147 & 49 & 59 & 169 & 175 & 168 & 103 & 104 & 148 & 178 & 111 & 70 \\
10 & 203 & 14 & 102 & 66 & 123 & 116 & 111 & 21 & 15 & 196 & 54 & 130 & 244 & 239 & 244 \\
196 & 118 & 21 & 164 & 34 & 129 & 100 & 230 & 170 & 7 & 247 & 118 & 79 & 59 & 79 & 221 \\
38 & 189 & 221 & 142 & 11 & 39 & 142 & 255 & 168 & 49 & 78 & 150 & 157 & 183 & 101 & 161 \\
145 & 139 & 227 & 131 & 17 & 224 & 116 & 99 & 108 & 144 & 176 & 161 & 50 & 35 & 105 & 20 \\
150 & 211 & 123 & 240 & 174 & 55 & 101 & 210 & 141 & 87 & 83 & 246 & 46 & 70 & 53 & 46 \\
108 & 46 & 80 & 112 & 172 & 232 & 228 & 69 & 97 & 232 & 166 & 102 & 70 & 63 & 94 & 18 \\
18 & 214 & 79 & 151 & 79 & 250 & 10 & 116 & 81 & 115 & 228 & 77 & 121 & 12 & 153 & 167 \\
131 & 133 & 132 & 246 & 53 & 94 & 132 & 39 & 151 & 183 & 207 & 36 & 194 & 222 & 227 & 70 \\
141 & 193 & 91 & 210 & 120 & 146 & 152 & 224 & 202 & 110 & 34 & 0 & 73 & 176 & 4 & 0 \\
198 & 2 & 215 & 20 & 141 & 203 & 183 & 92 & 214 & 217 & 37 & 140 & 141 & 161 & 211 & 248 \\
25 & 70 & 49 & 234 & 95 & 31 & 25 & 99 & 200 & 248 & 251 & 243 & 15 & 149 & 206 & 78 \\
116 & 135 & 103 & 157 & 37 & 64 & 242 & 116 & 246 & 219 & 17 & 71 & 249 & 157 & 127 & 34 \\
17 & 148 & 51 & 32 & 121 & 45 & 163 & 192 & 14 & 166 & 62 & 211 & 64 & 156 & 40 & 50 \\
220 & 210 & 244 & 208 & 41 & 113 & 132 & 254 & 115 & 174 & 22 & 231 & 196 & 188 & 67 & 126 \\
121 & 96 & 4 & 234 & 70 & 102 & 186 & 145 & 133 & 69 & 222 & 158 & 239 & 30 & 75 & 146
\end{array}\right]
$$

The entire plaintext, given by (3.1), is divided into 3 blocks, wherein each block is of size 16x16. In the 3rd block, we have appended 95 zeroes as characters, so that we make it a complete block. On using EK, FK, in the place of E and F, in the encryption algorithm, we carry out the encryption process 3 times, so that the complete plaintext is converted into the corresponding ciphertext. This ciphertext is given by (5.3).

The EK and FK are encrypted by using the E, the F, and applying the encryption algorithm. The resulting ciphertexts of the keys EK and FK are as follows, in (5.4) and (5.5).

These are transmitted to the receiver by the sender. In addition to these, the key bunch matrix E, the additional key matrix $\mathrm{F}$, the key $\mathrm{K}$ used in the processes permutation and substitution are sent by the sender to the receiver, in a secure manner. The number of additional characters appended in the last block is also informed to the receiver.

\begin{tabular}{|c|c|c|c|c|c|c|c|c|c|c|c|c|c|c|c|}
\hline 38 & 148 & 35 & 233 & 157 & 94 & 147 & 31 & 252 & 129 & 27 & 155 & 11 & 231 & 166 & 169 \\
\hline 227 & 99 & 59 & 188 & 89 & 55 & 67 & 212 & 75 & 208 & 216 & 147 & 227 & 18 & 217 & 166 \\
\hline 109 & 69 & 91 & 40 & 101 & 193 & 98 & 1 & 60 & 135 & 125 & 188 & 51 & 73 & 45 & 59 \\
\hline 24 & 125 & 41 & 75 & 17 & 36 & 157 & 134 & 53 & 226 & 20 & 204 & 25 & 248 & 99 & 200 \\
\hline 6 & 170 & 236 & 237 & 98 & 229 & 205 & 189 & 41 & 162 & 91 & 140 & 73 & 182 & 9 & 78 \\
\hline 212 & 221 & 116 & 182 & 225 & 53 & 203 & 180 & 252 & 188 & 235 & 233 & 247 & 88 & 149 & 86 \\
\hline 66 & 213 & 88 & 95 & 157 & 43 & 93 & 182 & 30 & 177 & 134 & 138 & 98 & 231 & 199 & 79 \\
\hline 241 & 156 & 13 & 75 & 4 & 115 & 91 & 86 & 228 & 10 & 138 & 18 & 244 & 149 & 8 & 0 \\
\hline 81 & 145 & 34 & 125 & 247 & 160 & 62 & 115 & 40 & 239 & 253 & 1 & 17 & 37 & 113 & 74 \\
\hline 116 & 83 & 51 & 136 & 116 & 57 & 107 & 126 & 236 & 164 & 167 & 104 & 18 & 200 & 83 & 18 \\
\hline 0 & 126 & 111 & 20 & 47 & 152 & 172 & 104 & 81 & 138 & 65 & 210 & 181 & 145 & 206 & 78 \\
\hline 53 & 44 & 149 & 99 & 233 & 58 & 76 & 209 & 40 & 33 & 42 & 224 & 179 & 221 & 160 & 90 \\
\hline 223 & 201 & 57 & 138 & 120 & 212 & 159 & 105 & 173 & 176 & 164 & 233 & 198 & 118 & 108 & 126 \\
\hline 134 & 55 & 93 & 84 & 231 & 232 & 250 & 150 & 28 & 201 & 170 & 51 & 8 & 112 & 254 & 139 \\
\hline 28 & 37 & 30 & 175 & 46 & 13 & 75 & 10 & 139 & 102 & 118 & 118 & 39 & 97 & 121 & 241 \\
\hline 44 & 104 & 224 & 190 & 16 & 209 & 148 & 231 & 150 & 83 & 22 & 252 & 166 & 156 & 19 & 203 \\
\hline
\end{tabular}




\begin{tabular}{|c|c|c|c|c|c|c|c|c|c|c|c|c|c|c|c|}
\hline 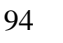 & 156 & 21 & 226 & 84 & 41 & 36 & 223 & 26 & 192 & 97 & 94 & 125 & 189 & 59 & 237 \\
\hline 231 & 99 & 144 & 43 & 241 & 159 & 157 & 217 & 34 & 186 & 20 & 244 & 191 & 43 & 85 & 152 \\
\hline - & 155 & 0 & 98 & 149 & 218 & 53 & 3 & 172 & 204 & 84 & 118 & 108 & 12 & 55 & 76 \\
\hline 151 & 99 & 60 & 175 & 243 & 251 & 17 & 35 & 228 & 141 & 243 & 153 & 49 & 174 & 240 & 181 \\
\hline 04 & 139 & 65 & 240 & 120 & 95 & 148 & 93 & 108 & 245 & 248 & 240 & 102 & 58 & 108 & o \\
\hline 0 & 96 & 157 & 14 & 62 & 24 & 190 & 164 & 167 & 227 & 8 & 251 & 109 & 45 & 97 & 187 \\
\hline 43 & 2 & 132 & 243 & 32 & 40 & 78 & 173 & 103 & 176 & 38 & 58 & 163 & 124 & 185 & 233 \\
\hline 242 & 73 & 171 & 62 & 202 & 177 & 208 & 202 & 3 & 213 & 221 & 121 & 115 & 38 & 96 & 154 \\
\hline 89 & 203 & 141 & 46 & 252 & 18 & 149 & 99 & 165 & 125 & 22 & 239 & 46 & 36 & 52 & 54 \\
\hline 21 & 175 & 143 & 164 & 45 & 196 & 2 & 163 & 2 & 80 & 208 & 112 & 229 & 196 & 97 & 56 \\
\hline 253 & 217 & 107 & 97 & 162 & 180 & 229 & 220 & 142 & 161 & 225 & 229 & 109 & 239 & 177 & 19 \\
\hline 98 & 92 & 205 & 89 & 31 & 151 & 4 & 68 & 116 & 9 & 171 & 217 & 100 & 53 & 247 & 110 \\
\hline 225 & 136 & 232 & 70 & 124 & 192 & 22 & 140 & 188 & 211 & 109 & 134 & 97 & 203 & 41 & 238 \\
\hline 51 & 123 & 20 & 89 & 179 & 222 & 63 & 93 & 70 & 92 & 203 & 170 & 185 & 35 & 52 & 90 \\
\hline 167 & 116 & 159 & 7 & 174 & 158 & 159 & 82 & 118 & 160 & 73 & 39 & 110 & 31 & 75 & 179 \\
\hline 26 & 247 & 244 & 28 & 163 & 166 & 11 & 144 & 203 & 86 & 180 & 33 & 200 & 138 & 201 & 181 \\
\hline 12 & 181 & 81 & 91 & 149 & 203 & 173 & 223 & 77 & 216 & 176 & 223 & 5 & 211 & 181 & 161 \\
\hline 39 & 123 & 8 & 93 & 233 & 6 & 230 & 76 & 127 & 189 & 226 & 144 & 34 & 83 & 134 & 221 \\
\hline 251 & 138 & 37 & 98 & 24 & 37 & 227 & 199 & 123 & 24 & 134 & 133 & 140 & 186 & 132 & 8 \\
\hline 114 & 170 & 231 & 225 & 178 & 141 & 116 & 190 & 89 & 63 & 243 & 59 & 200 & 61 & 84 & 247 \\
\hline 49 & 192 & 79 & 209 & 105 & 47 & 144 & 254 & 206 & 42 & 178 & 254 & 228 & 204 & 220 & 49 \\
\hline 9 & 167 & 2 & 213 & 179 & 134 & 249 & 23 & 193 & 100 & 28 & 205 & 62 & 69 & 119 & 91 \\
\hline 109 & 6 & 127 & 88 & 45 & 2 & 147 & 226 & 135 & 49 & 240 & 209 & 246 & 206 & 224 & 125 \\
\hline 71 & 185 & 146 & 5 & 72 & 206 & 99 & 67 & 233 & 152 & 192 & 253 & 13 & 154 & 215 & 36 \\
\hline 67 & 22 & 233 & 65 & 248 & 236 & 180 & 223 & 114 & 45 & 4 & 195 & 106 & 215 & 123 & 135 \\
\hline 0 & 31 & 236 & 22 & 169 & 81 & 206 & 62 & 34 & 170 & 194 & 54 & 77 & 233 & 160 & 141 \\
\hline 153 & 196 & 90 & 225 & 27 & 31 & 225 & 226 & 94 & 179 & 143 & 130 & 195 & 44 & 64 & 82 \\
\hline 255 & 30 & 13 & 203 & 62 & 194 & 21 & 17 & 106 & 201 & 56 & 2 & 71 & 210 & 24 & 231 \\
\hline 17 & 119 & 167 & 107 & 156 & 63 & 62 & 233 & 182 & 46 & 160 & 38 & 58 & 50 & 165 & 173 \\
\hline 78 & 175 & 80 & 75 & 113 & 233 & 225 & 172 & 42 & 176 & 15 & 42 & 1 & 132 & 238 & 95 \\
\hline 144 & 42 & 54 & 3 & 190 & 173 & 131 & 50 & 50 & 200 & 229 & 128 & 161 & 103 & 47 & 37 \\
\hline 85 & 203 & 59 & 113 & 31 & 245 & 244 & 190 & 62 & 39 & 37 & 176 & 196 & 123 & 66 & 129 \\
\hline
\end{tabular}

The cryptanalysis carried out in this investigation strongly indicate that this cipher is a potential one and it can be applied for the transmission of text of any size and gray level/color images.

\begin{tabular}{|c|c|c|c|c|c|c|c|c|c|c|c|c|c|c|c|}
\hline 2 & 95 & 7 & 118 & 130 & 53 & 132 & 123 & 10 & 29 & 82 & 113 & 143 & 155 & 35 & 59 \\
\hline 70 & 109 & 146 & 221 & 38 & 152 & 190 & 52 & 18 & 202 & 30 & 202 & 95 & 137 & 241 & 108 \\
\hline 7 & 166 & 2 & 21 & 200 & 105 & 36 & 1 & 63 & 251 & 128 & 219 & 29 & 149 & 6 & 185 \\
\hline 222 & 220 & 147 & 41 & 44 & 159 & 82 & 136 & 151 & 205 & 190 & 65 & 210 & 146 & 172 & 107 \\
\hline 30 & 241 & 25 & 80 & 94 & 28 & 65 & 18 & 241 & 112 & 50 & 238 & 98 & 5 & 204 & 255 \\
\hline 42 & 149 & 71 & 197 & 104 & 106 & 49 & 71 & 180 & 154 & 87 & 213 & 137 & 97 & 152 & 210 \\
\hline 5 & 148 & 246 & 66 & 203 & 164 & 188 & 168 & 232 & 105 & 222 & 143 & 61 & 40 & 255 & 81 \\
\hline 89 & 177 & 154 & 111 & 224 & 201 & 213 & 213 & 14 & 88 & 184 & 245 & 226 & 74 & 58 & 179 \\
\hline 148 & 70 & 31 & 251 & 7 & 1 & 126 & 100 & 11 & 249 & 244 & 73 & 240 & 58 & 212 & 23 \\
\hline 174 & 150 & 143 & 158 & 163 & 155 & 137 & 239 & 139 & 96 & 130 & 82 & 244 & 105 & 87 & 23 \\
\hline 1 & 63 & 184 & 102 & 93 & 113 & 70 & 156 & 185 & 241 & 173 & 157 & 58 & 15 & 216 & 17 \\
\hline 202 & 1 & 47 & 213 & 55 & 48 & 231 & 37 & 13 & 38 & 35 & 19 & 157 & 40 & 21 & 68 \\
\hline 61 & 71 & 255 & 44 & 109 & 131 & 140 & 98 & 182 & 14 & 95 & 176 & 239 & 38 & 129 & 113 \\
\hline 111 & 235 & 208 & 238 & 192 & 46 & 187 & 5 & 63 & 75 & 131 & 23 & 114 & 114 & 204 & 75 \\
\hline 49 & 230 & 221 & 242 & 247 & 131 & 6 & 142 & 45 & 198 & 167 & 221 & 227 & 106 & 129 & 8 \\
\hline 85 & 3 & 99 & 170 & 211 & 133 & 6 & 225 & 234 & 109 & 187 & 240 & 237 & 200 & 53 & 139 \\
\hline
\end{tabular}


and

$\begin{array}{llllllllllllllll}91 & 46 & 145 & 165 & 147 & 49 & 59 & 169 & 175 & 168 & 103 & 104 & 148 & 178 & 111 & 70 \\ 10 & 203 & 14 & 102 & 66 & 123 & 116 & 111 & 21 & 15 & 196 & 54 & 130 & 244 & 239 & 244 \\ 196 & 118 & 21 & 164 & 34 & 129 & 100 & 230 & 170 & 7 & 247 & 118 & 79 & 59 & 79 & 221 \\ 38 & 189 & 221 & 142 & 11 & 39 & 142 & 255 & 168 & 49 & 78 & 150 & 157 & 183 & 101 & 161 \\ 145 & 139 & 227 & 131 & 17 & 224 & 116 & 99 & 108 & 144 & 176 & 161 & 50 & 35 & 105 & 20 \\ 150 & 211 & 123 & 240 & 174 & 55 & 101 & 210 & 141 & 87 & 83 & 246 & 46 & 70 & 53 & 46 \\ 108 & 46 & 80 & 112 & 172 & 232 & 228 & 69 & 97 & 232 & 166 & 102 & 70 & 63 & 94 & 18 \\ 18 & 214 & 79 & 151 & 79 & 250 & 10 & 116 & 81 & 115 & 228 & 77 & 121 & 12 & 153 & 167 \\ 131 & 133 & 132 & 246 & 53 & 94 & 132 & 39 & 151 & 183 & 207 & 36 & 194 & 222 & 227 & 70 \\ 141 & 193 & 91 & 210 & 120 & 146 & 152 & 224 & 202 & 110 & 34 & 0 & 73 & 176 & 4 & 0 \\ 198 & 2 & 215 & 20 & 141 & 203 & 183 & 92 & 214 & 217 & 37 & 140 & 141 & 161 & 211 & 248 \\ 25 & 70 & 49 & 234 & 95 & 31 & 25 & 99 & 200 & 248 & 251 & 243 & 15 & 149 & 206 & 78 \\ 116 & 135 & 103 & 157 & 37 & 64 & 242 & 116 & 246 & 219 & 17 & 71 & 249 & 157 & 127 & 34 \\ 17 & 148 & 51 & 32 & 121 & 45 & 163 & 192 & 14 & 166 & 62 & 211 & 64 & 156 & 40 & 50 \\ 220 & 210 & 244 & 208 & 41 & 113 & 132 & 254 & 115 & 174 & 22 & 231 & 196 & 188 & 67 & 126 \\ 121 & 96 & 4 & 234 & 70 & 102 & 186 & 145 & 133 & 69 & 222 & 158 & 239 & 30 & 75 & 146\end{array}$

\section{REFERENCES}

[1] Dr.V.U.K.Sastry, K.Shirisha, "A Novel Block Cipher Involving a Key Bunch Matrix and a Key-based Permutation and Substitution", in International Journal of Advanced Computer Science and Applications(IJACSA), Vol. 3, No. 12, Jan 2012, pp.16-122.

[2] William Stallings: Cryptography and Network Security: Principle and Practices", Third Edition 2003, Chapter 2, pp. 29.

\section{AUTHORS PROFILE}

Dr. V. U. K. Sastry is presently working as Professor in the Dept. of Computer Science and Engineering (CSE), Director (SCSI), Dean (R \& D), SreeNidhi Institute of Science and Technology (SNIST), Hyderabad, India. He was Formerly Professor in IIT, Kharagpur, India and worked in IIT,
Kharagpur during 1963 - 1998. He guided $14 \mathrm{PhDs}$, and published more than 87 research papers in various International Journals. He received the Best Engineering College Faculty Award in Computer Science and Engineering for the year 2008 from the Indian Society for Technical Education (AP Chapter), Best Teacher Award by Lions Clubs International, Hyderabad Elite, in 2012, and Cognizant- Sreenidhi Best faculty award for the year 2012. His research interests are Network Security \& Cryptography, Image Processing, Data Mining and Genetic Algorithms.

K. Shirisha is currently working as Associate Professor in the Department of Computer Science and Engineering (CSE), SreeNidhi Institute of Science \& Technology (SNIST), Hyderabad, India, since February 2007. She is pursuing her Ph.D. Her research interests are Information Security and Data Mining. She published 9 research papers in International Journals. She stood University topper in the M.Tech.(CSE). 